\title{
Testing the Morphological Aspects of Polyethylene Glycol-primed Tomato Seeds with Proportional Odds Analysis
}

\author{
A. Liptay and N. Zariffa ${ }^{1}$ \\ Research Station, Agriculture Canada, Harrow, Ont. NOR 1G0, Canada
}

Additional index words. germination, osmoticum

\begin{abstract}
Priming tomato (Lycopersicon esculentum Mill) seeds in aerated -0.5 MPa polyethylene glycol (PEG) enhanced the emergence rate and the extent and percentage of embryo radicles protruding partially or completely through the seed endosperm. The radicles' growth, however, was arrested at the seedcoat. The time course of radicle protrusion through the endosperm of seeds in PEG for the first 24 hours paralleled that of seeds germinating in aerated water; however, radicle protrusion continued through the seedcoats of seeds germinating in water. The radicle of the high-vigor PI-341988 tomato line protruded more rapidly through the endosperm than that of the low-vigor ST-24 line.
\end{abstract}

Priming seeds in an osmoticum such as polyethylene glycol (PEG) often is performed in the seed industry to enhance the onset of

Received for publication 30 Nov. 1992. Accepted for publication 22 Apr. 1993. The cost of publishing this paper was defrayed in part by the payment of page charges. Under postal regulations, this paper therefore must be hereby marked advertisement solely to indicate this fact.

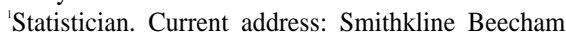
Pharmaceuticals, Presbyterian Medical Center, Clinical Research Unit, 51 North 39th St., Philadelphia, PA 19104. seed germination after sowing. In tomato seeds, the endosperm cap and testa form a barrier that the radicle must penetrate before germination can occur. Physically removing this barrier reduces germination time (Dahal et al., 1990, Groot et al., 1988; Liptay and Schopfer, 1983). Thus, the endosperm bars germination and helps control germination rate and vigor (Liptay and Schopfer, 1983; Liptay and Tan, 1985). Groot et al. (1988) found that gibberellin weakened a tomato seed's endospem.

The purpose of the present study was to quantify the physical changes, morphological changes, or both associated with priming tomato seeds with PEG and compare the response of high- and low-vigor seeds to priming.

Tomato seeds were primed either in an aerated solution of $-5 \mathrm{MPa}$ PEG 6000 or water at $22 \mathrm{C}$ in 2-liter graduated cylinders that were covered with parafilm (American National, Greenwich, Corm.). The tomato lines used were those reported in earlier work: PI-341988, a high-vigor line, and ST-24, a low-vigor line (Liptay and Schopfer, 1983; Liptay and Tan, 1985). PEG solutions were prepared according to Michel and Kaufman (1973). Fifty seeds were sampled from each of the two lines and two priming treatments, i.e., with or without PEG, at 2,24,48,72, and $96 \mathrm{~h}$. The 2-h time course represented the seed's initial state because the dry seeds were too difficult to cut precisely. Fifty seeds from each treatment were cut along the longitudinal axis. The excision was not always perfectly aligned; therefore, the extent to which the radicle protruded through the endosperm could not be discerned in all samples. Thus, the data are the means of only those seeds in which endosperm degradation could be discerned easily when viewed in the scanning electron microscope (SEM) (model S510; Hitachi, Tokyo). The number of seeds that could be assessed properly per priming treatment varied from 31 to 50 . The cut seeds were prepared for the SEM by freezing them on dry ice, freeze-drying them for $24 \mathrm{~h}$, and attaching them with silver paste to stubs and gold-sputtering their cut surfaces.

Emergence was measured on an ordinal 


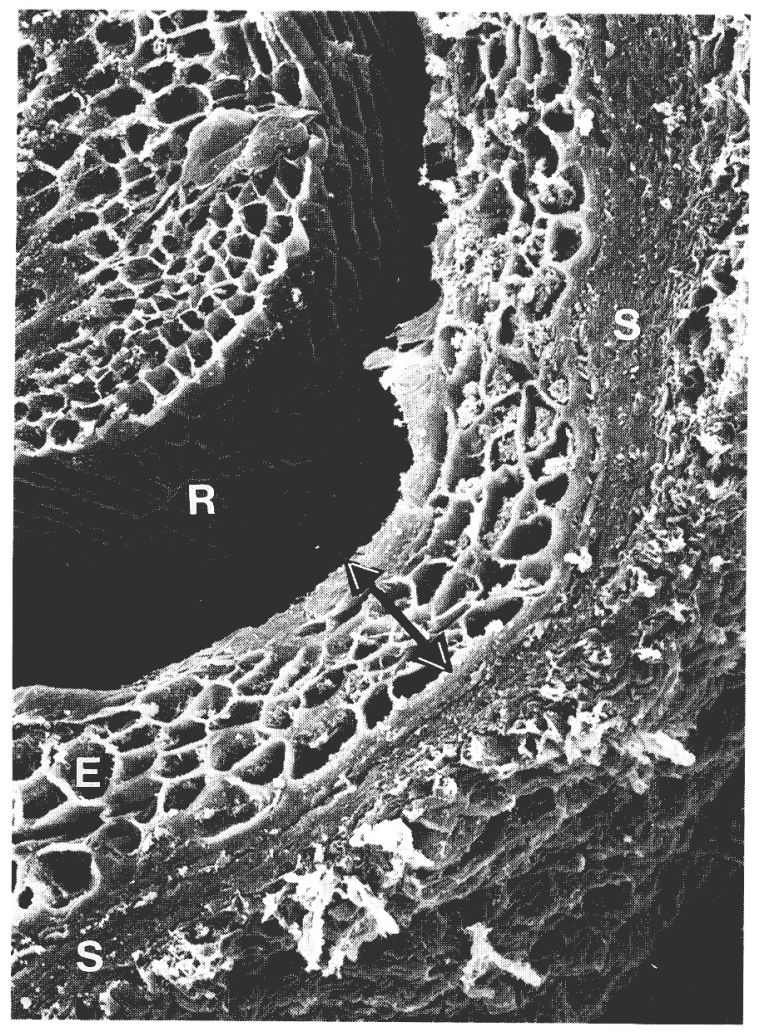

Fig. 1. Scanning electron microscope $(\mathrm{SEM})$ micrograph $(\times 27)$ of a longitudinal section along the flat plane of a tomato seed $2 \mathrm{~h}$ after priming with polyethylene glycol began. $\mathrm{R}=$ radicle, $\mathrm{E}=$ endosperm, and $\mathrm{S}$ = seedcoat. The double-ended arrow indicates the compression zone of the endosperm by the radicle during priming. The rating for this particular specimen was $0 \%$ penetration of the endosperm by the radicle. The seeds were prepared for SEM viewing as outlined in the text.

scale as follows: seeds with radicles not protruding into the endosperm, seeds with radicles protruding from $0 \%$ to $50 \%$, seeds with radicles protruding from $0 \%$ to $100 \%$ (completely compressed endosperms), and seeds with totally emerged radicles. The latter category could not be included in the analysis, as no radicles emerged under PEG priming. This result led to a confounding of the above factors.

Data were recorded as a numerical score and tested using analysis of variance. Such an approach assumes a normal distribution, which was not met in this case. A second approach is to consider the data as purely categorical and use an appropriate log linear model (McCullagh and Nelder, 1989). Such models require more factors, as they do not use the information contained in the ordinal scale. Recent models, such as McCullagh's (1980) proportional odds analysis, are directed at ordinal categorical data. McCullagh's model has a desirable invariance property with respect to pooling ordinal categories arising from an implicit continuous scale. This model also is readily available in PROC LOGISTIC and PROC PROBIT, version 6 (SAS Institute, Cary, N.C.).

In our case, the model is defined as $\log \left(\gamma_{j}\right)$ $\left.1-\gamma_{\mathrm{j}}\right)=\theta_{\mathrm{j}}-\beta^{\prime} \mathrm{x}$, where $\mathrm{j}=1,2, \gamma_{1}=\pi_{1}, \gamma_{2}=\pi_{1}$ $+\pi_{2}, \gamma_{3}=\pi_{1}+\pi_{2}+\pi_{3}, \quad$ is the multinominal probability attached to category $\mathrm{j}$, and $\beta$ and $\theta$ are the parameter vectors we wish to estimate. The $\theta$ 's are of no intrinsic interest they represent the logit of cumulative response when $\mathrm{x}=$
0 . The $\theta$ js often are referred to as cutoff points. Also, one of the assumptions this model makes is that the logit cumulative probability curves are parallel. The b s are the parameters through which we establish the significance of the explanatory variables, $\mathrm{x}$.

The analysis was comprised of two cultivars, PEG priming (with or without), germination time $(2,24,48,72$, and $96 \mathrm{~h})$, and extent of endosperm protrusion by the radicle $(0 \%$, O $\%$ to $50 \%$, or $\mathrm{O} \%$ to $100 \%$ ).

Longitudinally cut seeds were viewed with the SEM to establish the time course of endosperm protrusion by the radicle during PEG priming (Fig. 1). In ST-24 seeds, the percentage of radicles not penetrating the endosperm was similar in the aerated water and PEG (Fig. 2). $\mathrm{AH}$ other similar comparisons exhibited differences for both tomato lines. Thus, $>50 \%$ of PEG-primed ST-24 seeds and $\approx 75 \%$ of PI341988 seeds had their radicles protruding at least halfway through the endosperm $96 \mathrm{~h}$ after priming began. Moreover, in PI-341988 seeds, the endosperm was penetrated earlier by the radicle than in ST-24 seeds.

Initial models included all two-way interaction terms and main-effect terms for cultivar, PEG priming, and time. Interaction terms not significant at $P \leq 0.05$ were removed. The final model included all main effects and a PEG $\mathrm{x}$ time interaction term (Table 1).

The assumption of proportional odds $\left[\mathrm{x}_{(9)}=\right.$ 6.2702, $P \leq 0.7126$ ] was met, and the likeli-

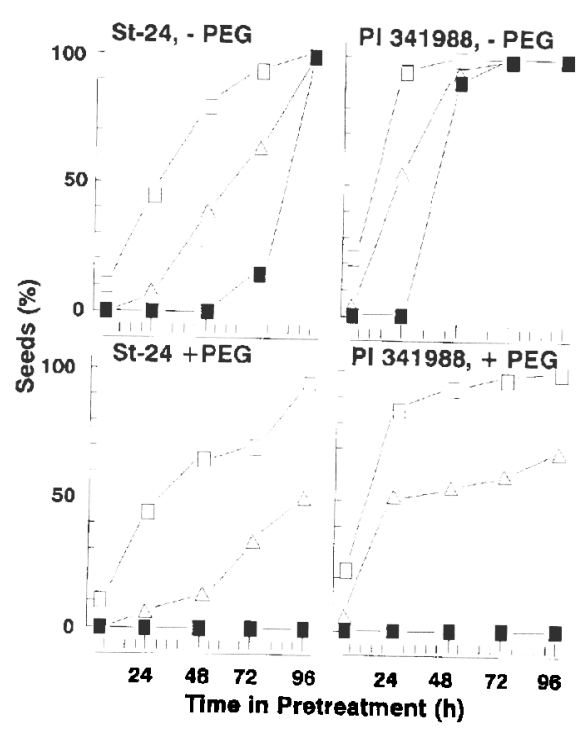

Fig. 2. Percentage distribution of seeds with radicles protruding from $0 \%$ to $50 \%$ into the endosperm ( $\square)$, radicles protruding from $0 \%$ to $100 \%$ into the endosperm (completely compressed endosperms and seeds) $(\Delta)$, and with emerged radicles ( $\mathbf{0}$ ). ST-24 or PI-341988 tomato seeds were primed either in aerated -5 MPa polyethylene glycol or distilled water. Seeds were sampled at 2, 24, 48, 72, and $96 \mathrm{~h}$ from the beginning of priming.

Table 1. Proportional odds analysis of tomato seed radicle protrusion into the endosperm.

\begin{tabular}{lcr}
\hline \hline Parameter & $\mathrm{df}$ & $\mathrm{x}^{2}$ \\
\hline Cultivar & 882 & 83.3 \\
Polyethylene glycol (PEG) & & \\
$\quad$ (with or without) & 1 & 21.5 \\
Time (T) & 1 & 131.4 \\
$\mathrm{~T} \times$ PEG & $3^{2}$ & 25.3 \\
$\theta_{1}$ & 1 & 137.5 \\
$\theta_{2}$ & 1 & 35.6 \\
\hline${ }^{2}$ Only three available df & &
\end{tabular}

${ }^{2}$ Only three available df.

hood ratio test for goodness of fit indicated no gross departure from the model $\left[\mathrm{x}_{(23)}^{2}=\right.$ 29.6717, $P \leq 0.1591]$.

The effect of the cultivar was independent of time and PEG priming, with a cumulative odds ratio ofe' $9=6.7$, a result indicating that emergence was associated with cultivar. PI341988 had higher cumulative probability of emergence than ST-24. The effect of PEG vs. time was not independent (Fig. 2).

Endosperm degradation and radicle protrusion up to but not through the tests seemed to be the main effect of seed priming (Fig. 2). These morphological results support work by Ellis and Butcher (1988), who reported that the main effect of priming seeds was a reduced thermal germination time. The radicle's penetration through the endosperm is the most difficult aspect of initial germination stages. The results demonstrate cultivar differences, even in response rate to PEG priming.

\section{Literature Cited}

Dahal, P., K. Bradford, and R.A. Jones. 1990. Effects of priming and endosperm integrity on seed germination rates of tomato genotypes. J. Expt. Bot. 41:1431-1439. 
Ellis, R.H. and P.D. Butcher. 1988. The effects of priming and "natural" differences in quality amongst onion seed lots on the response of the rate of germination to temperature and the identification of the characteristics under genotypic control. J. Expt. Bot. 39:939-950.

Grout, S.P.C., B. Kieliszewska-Robicka, E. Vermeer, and C.M. Karssen. 1988. Gibberellin-induced hydrolysis of endosperm cell walls in gibberel- lin-deficient tomato seeds prior to radicle protrusion. Planta 174:500-504.

Liptay, A. and P. Schopfer. 1983. Effect of water stress, seed coat restraint and abscisic acid upon different germination capabilities of two tomato lines at low temperature. Plant Physiol. 73:935-938.

Liptay, A. and C.S. Tan. 1985. Effect of available water on germination of polyethylene glycol (PEG) pretreated or untreated tomato seeds. J.
Amer. Soc. Hort Sci. 110:748-751.

McCullagh, P.M. 1980. Regression models for ordinal data. J. Royal Stat. Sot. series B. 42:109-142.

McCullagh, P.M. and J.A. Nelder. 1989. Generalized linear models. 2nd ed. Chapman and Hall, New York.

Michel, B.E. and M.R. Kaufman. 1973. The osmotic potential of polyethylene glycol 6000. Plant Physiol. 51:914-916. 